weile extensiven Schutzbereichsinterpretation. ${ }^{32}$ Sieht man die Definitionskompetenz bezüglich dessen, was Religionsausübung ist, in erster Linie beim Grundrechtsträger, so muss damit eine angemessene Schrankenregelung einhergehen. Mit der Anerkenntnis des Art. I 36 I WRV als Schranke hätte sich das Gericht auf der Seite einer zunehmenden Zahl an Befürwortern befunden. ${ }^{33}$

\title{
Fazit
}

Die Entscheidung mag zwar im Ergebnis überzeugen. Die Begründung aber ist undogmatisch. Das Grundgesetz gewährleistet Grundrechte mit differenzierter Schutzbereichs- und Schrankenregelung. Diese Abstufungen ebnet das BVerfG mit dieser Entscheidung ein. Das Abweichen von der parallelen Anwendbarkeit einschlägiger Grundrechte lässt sich nicht nach Maßgabe der Meist-Betroffenheit rechtfertigen, nicht wenn es sich um schrankendivergierende Grundrechte handelt, und erst recht nicht, wenn gar keine vorrangige Grundrechtsbetroffenheit gegeben ist. Konsequenz dieser Rechtsprechung ist auch, dass derjenige, der sich gleichzeitig auf die Religionsfreiheit und auf ein unter Vorbehalt stehendes Grundrecht berufen kann, letztlich weniger geschützt ist, als wenn ihm nur die Religionsfreiheit zur Seite steht. In diesem Fall besteht gar nicht erst die Möglichkeit einer Grundrechtsverdrängung, so dass die (nach Ansicht des BVerfG) schrankenlos gewährte Religionsfreiheit voll und nicht nur verstärkend - wirkt. Die Problematik des Schächtens entzündet sich nicht an Fragen des Schutzbereichs oder der Grundrechtskonkurrenzen, sondern auf der Ebene der Grundrechtsschranken. Das sollte anerkannt werden, indem Lösungen an genau diesem Punkt ansetzen. Die Anerkenntnis des Art. I 36 I WRV als Schrankenregelung wäre ehrlicher und klarer gewesen.

\section{Benno Kirsch Verbrechensbekämpfung durch private Sicherheitsdienste?}

\section{Public-Private-Partnership der »Inneren Sicherbeit"}

Zwei Behauptungen führen die Befürworter der Privatisierung öffentlicher Sicherheit immer wieder ins Feld: dass zum einen Kriminalität immer weiter ansteige - verbunden häufig mit der Behauptung, dass insbesondere die Jugendkriminalität zunehme und die Täter immer brutaler würden - und dass der Staat mit dem Problem überfordert sei, während andererseits die privaten Sicherheitsdienste sich inzwischen als zuverlässiger Bestandteil des "Systems Innerer Sicherheit« etabliert hätten. In seinem berühmten Vortrag hat Hoffmann-Riem die Befürchtung geäußert, mit den privaten Sicherheitsdiensten könne ein demokratisch nicht zu kontrollierendes Macht- und Gewaltpotential entstehen und den Charakter der Jedermannrechte, auf die sie sich berufen, durch die professionelle Inanspruchnahme derselben in ihrem Wesen verändern (Hoffmann-Riem I977). Tempi passati. Die nicht intendierte Folge

32 Ausführlich hierzu Müller-Volbehr, Das Grundrecht der Religionsfreiheit und seine Schranken, DÖV I995, 30I (30I ff.), sowie Kästner (Fn. 8), S. 979, der hier von Hypertrophierung spricht.

33 Überzeugend mit weiteren Argumenten Muckel, Religiöse Freiheit und staatliche Letztentscheidung, Habil., Berlin I997, S. 224 ff., m. w. N. 
seines Vortrags war, dass im Verlauf der Jahre alle möglichen Anstrengungen unternommen wurden, Hoffmann-Riems Behauptungen zu widerlegen (vgl. Mahlberg 1988, Bueß 1997, Schnekenburger 1999), was überzeugend gelungen ist, denn er versucht lediglich, politisches Ressentiment in juristische Kategorien zu übertragen. Die Frage ist nicht mehr, ob sich private Sicherheitsdienste etabliert haben, sondern wie weit man sie in die staatliche Sicherheitsgewährleistung einbeziehen will.

Auf diesem Weg ist man in den letzten Jahren ein gutes Stück vorangekommen, man denke nur an die Änderung des $\ 34 \mathrm{a}$ GewO durch das Verbrechensbekämpfungsgesetz I 994 oder die geplante gesetzliche Regelung des Sicherheitsgewerbes durch die gegenwärtige Bundesregierung, in dem sie »den rechtlichen Rahmen... zu präzisieren « gedenkt (Schily 2000: 6). Was genau in diesem Gesetz »präzisiert« werden soll, bleibt allerdings im Dunkeln, denn die Rechtslage ist eindeutig. Der Verlauf der Diskussion in Zeitschriften, auf Tagungen, Seminaren und Kongressen lässt darauf schließen, dass eher eine Ausweitung der Aufgaben und Befugnisse angestrebt wird als eine Beschränkung im Sinne Hoffmann-Riems. Das Gewerbe selbst indes lehnt eine gesetzliche Regelung ab (vgl. BDWS 2000).

Der Ausgang des Gesetzgebungsverfahrens ist gegenwärtig ungewiss, aber es bietet einen Anlass zu spekulieren, welche Möglichkeiten die Privatisierung der Verbrechenskontrolle bietet. Heißt es nicht allenthalben, dass Private sämtliche Aufgaben billiger und besser erfüllen könnten als der Staat? Warum sollte also ausgerechnet die Aufgabe der Verbrechensbekämpfung einer (Teil-)Privatisierung entzogen werden? Vielleicht kann das Gewerbe im Rahmen einer Partnerschaft mit der Polizei unterstützend bei der Verbrechenskontrolle mitwirken? Vielleicht ergeben sich durch die Integration privater Sicherheitsdienste in die polizeiliche Verbrechensbekämpfung Synergieeffekte, die nicht zu nutzen geradezu sträflich wäre?

Im Folgenden werden anhand dreier kleiner Fallstudien, die im Rahmen eines größeren Projektes angefertigt wurden, die Möglichkeiten der Einbeziehung Privater in die staatliche Verbrechensbekämpfung geprüft und das Potenzial der Privaten zur von beiden Seiten - des Gewerbes und, nach inzwischen weitgehend ausgeräumten Bedenken, der Polizei - heftig gewünschten Zusammenarbeit mit der Polizei ausgelotet. Zuerst werde ich darstellen, ob und in welcher Weise bereits heute die Rechtsordnung die Beteiligung Privater an der Verbrechensbekämpfung ermöglicht (2.). Sodann berichte ich von meiner empirischen Erforschung der Tätigkeit von Wachleuten im öffentlichen Personennahverkehr (ÖPNV). Es handelt sich hierbei um ein Feld, das gesteigerte öffentliche Aufmerksamkeit genießt und zu ganz gegensätzlichen Bewertungen verleitet. Während der einen Sicherheitsgefühl durch den Anblick der Wächter steigt, sehen die anderen in ihnen bloß verkappte Schläger, die Jagd auf Obdachlose machen (3.).

Zum Zweiten untersuche ich anhand der polizeilichen Videoüberwachung in der Stadt Leipzig die Auswirkungen der Bereitstellung von Sicherheitstechnik durch die Industrie auf die Polizeiarbeit und ihre Auswirkungen auf die Bevölkerung (4.). Drittens gehe ich in einem eher theoretisch-spekulativen Teil auf die Urform privater Sicherheitsdienste, den Werkschutz, und seinen Beitrag zur Herstellung von Sicherheit über die betrieblichen Grenzen hinaus ein. In seiner klassischen Form als Sicherheitsdienst für einen für den Publikumsverkehr weitgehend abgeschotteten Bereich gedacht, lassen sich unter Umständen die Chancen und Risiken der Privatisierung der Verbrechensbekämpfung am deutlichsten, weil wie unter Laborbedingungen beobachtet, herausschälen (5.). Und schließlich versuche ich, die Konsequenzen für die Arbeit der Polizei zu ermessen, die sich aus der Kooperation mit den Privaten ergeben (6.). 
So eindeutig scheint sich die Frage nach den Möglichkeiten zur Beteiligung Privater an der Strafverfolgung nicht beantworten zu lassen. Wohl wird Verbrechensbekämpfung von den Gerichten als eine Hoheitsaufgabe verstanden, die sich deshalb der Privatisierung entzieht, und entsprechend sind diesbezügliche Bestrebungen in der Vergangenheit immer wieder gescheitert. An einem unscheinbaren, aber erhellenden Beispiel lässt sich zeigen, wie gering die rechtlichen Spielräume für die private Bekämpfung von Straftaten sind. In Berlin hatte ein mit der Parkraumbewirtschaftung beauftragter Privater einen Autofahrer beim Falschparken ertappt und die entsprechenden Daten der zuständigen Behörde übermittelt, die daraufhin einen Bußgeldbescheid an den Fahrer schickte. Das Amtsgericht stellte jedoch fest, dass dieses Vorgehen verfassungswidrig sei, weil »die Feststellung von Ordnungswidrigkeiten im Rahmen der Verkehrsüberwachung... eine typische Hoheitsaufgabe [ist], die zum Kernbereich staatlicher Verwaltung gehört«. Die Sanktionierung von Ordnungswidrigkeiten sei eine Form der Strafverfolgung, die lediglich ein niedrigeres Strafmaß als bei Strafprozessen erwarten lasse und deshalb nicht von »selbständig und eigenverantwortlich « handelnden »Zeugen« durchzuführen sei (AG Berlin-Tiergarten I996; vgl. KG Berlin I996).

Verbrechensbekämpfung wird von den Gerichten also als Staatsaufgabe angesehen, die nicht privatisierungsfähig ist, weil sie zu den Kernaufgaben des Staates gezählt wird, aus denen er seine Existenzberechtigung ableitet. Das Gewaltmonopol äußere sich vor allem in der Aufgabe der Wahrung des Rechtsfriedens und der Gleichheit aller Bürger vor dem Gesetz, weshalb »an die Stelle der Formel vom staatlichen Gewaltmonopol eigentlich eher das Wort vom staatlichen Rechtsmonopol« treten sollte (Scholz i983: 707).

Hier wird im Grunde aber bereits die Ansicht Stobers, eines vehementen Befürworters der Integration des Bewachungsgewerbes, vorbereitet, der das Gewaltmonopol sowohl unter Hinweis auf die Letztverantwortlichkeit des Staates wahren als auch differenziert schwächen will. Das Gewaltmonopol - verstanden als Monopol des Staates zur Strafverfolgung - wird von ihm zwar nicht zur Disposition gestellt, weil es »ein essentieller und unverzichtbarer Bestandteil der staatlichen Daseinssicherung und Zukunftsvorsorge « ist (Stober 1997: 890). Allerdings wird diese Sicht von Stober und den Verfechtern der Privatisierung wieder relativiert, indem sie zwar die Strafverfolgung als exklusive Staatsaufgabe begreifen, aber die Gefahrenabwehr keinesfalls. Hier sehen sie einen Handlungsspielraum, Private stärker einzubinden und relativieren das Gewaltmonopol dann doch. Polizeiaufgaben seien, so Stober, ohnehin nie statisch gemäß unverrückbaren Prinzipien festgelegt gewesen, sondern dem Wandel der Vorstellungen über das unterworfen, was Polizei sei (ebd.: 89I). Insofern könne man ruhig überlegen, welche Bereiche bestehender Staatsaufgaben auch im Sicherheitsbereich privatisiert werden könnten. Es komme lediglich auf die konkrete Ausgestaltung an. Also »können auch staatliche Gewaltaufgaben kooperativ erfüllt werden, sofern der Staat seiner Gewährleistungs-, Regulierungs- und Kontrollverantwortung ausreichend nachkommt. Deshalb kann man jenseits eines nicht abschließend beschreibbaren Kerns an polizeilicher Grundversorgung auch über Lockerungen des staatlichen Gewaltmonopols nachdenken.« (ebd.: 893) Wer angesichts der Integration der Privaten die Befürchtung äußert, sie könne negative Folgen für die unparteiische Sicherheitsgewährleistung durch die Polizei haben, wird von Stober eines Besseren belehrt: »Staatliches Gewaltmonopol, Unternehmer- und Bürgerverantwortung, « lautet sein Ceterum censeo, »stehen also nicht isoliert nebeneinander. Vielmehr können auch staatliche Gewaltaufgaben kooperativ erfüllt wer- 
den, sofern der Staat seiner Gewährleistungs-, Regulierungs- und Kontrollverantwortung ausreichend nachkommt.«(Stober I998: S. 3 I)

Die Frage, ob man private Sicherheitsdienste an der staatlichen Verbrechensbekämpfung teilhaben lassen darf, wird von manchen also zumindest offen gehalten. Sie haben, so darf vermutet werden, ihre Gründe. Insbesondere Rolf Stober (Universität Hamburg) und Andreas Peilert (Fachhochschule des Bundes für öffentliche Verwaltung, Fachbereich Bundesgrenzschutz) haben sich in dieser Richtung exponiert und sind bestrebt, das Bewachungsgewerbe aufzuwerten und Wissenschaft, Polizei und Wirtschaft für dieses Ziel zu gewinnen. Dabei bedienen sie sich durchaus anrüchiger Methoden: Stober etwa ist seit 1999 Chef der von ihm ins Leben gerufenen »Forschungsstelle Sicherheitsgewerbe (FORSI) an der Universität Hamburg, die von dem Sicherheitsunternehmen Securitas finanziert wird (vgl. FAZ 26. Mai 2000). Und Peilert, der Mitglied des wissenschaftlichen Beirates von FORSI ist, berichtet über das Institut in einer Tageszeitung, als habe er keine Verbindungen dorthin (vgl. Die Welt I 8. August 1999). Stober indes betont, dass seine wissenschaftliche Unabhängigkeit und die der dort forschenden Doktoranden gewährleistet sei. Aber es gehört nicht viel Phantasie dazu, anzunehmen, dass es sich bei FORSI um einen jener Thinktanks handelt, mit denen das politische Programm des Neoliberalismus in wissenschaftliche Begriffe gekleidet werden und über vermeintlich interessenlose Akteure wie die Polizei, Journalisten und Wissenschaftler in die Gesellschaft hinein getragen werden soll (vgl. Dixon 2000; Wacquant 2000). Es ist darüber hinaus bezeichnend, dass die Diskussion über Privatisierung, insbesondere der Inneren Sicherheit, vor allem von den »Vertreter[n] der säkularisierten Theologie der Herrschaft, nämlich der Jurisprudenz« (Haupt/Narr 1978: I86), geführt wird. Man fragt kaum mehr, was wünschenswert, sondern bloß noch, ob es zulässig ist. Es hat den Anschein, als habe man aufgegeben, sich inhaltlich über die Zukunft der Gewährung von Sicherheit auseinanderzusetzen, als wäre man sich grundsätzlich einig.

\section{Wachleute im Berliner ÖPNV}

Um nicht der Faszination der Abstraktion zu erliegen und in der Folge das politische Moment zu verkennen, das den Phänomenen innewohnt, habe ich eine Feldstudie in Berlin und Frankfurt a. M. durchgeführt, in der ich das Netzwerk Innere Sicherheit dieser beiden Städte analysiert habe. Mir ging es darum, die Funktion der privaten Sicherheitsdienste zu beschreiben und herauszubekommen, wie sich der Einsatz der Privaten - gesetzt, dass sich seit nicht langer Zeit eine neue Dimension ihrer Tätigkeit im öffentlichen Raum geöffnet hat - auf die gesamte Sicherheitsarchitektur im Mikrokosmos der genannten Städte auswirkt. Vielleicht, so meine Vermutung, könnten sich Veränderungen auf Seiten des Gewaltmonopols feststellen lassen, die auf eine Zusammenarbeit mit den Privaten zurückzuführen ist. Ich habe deshalb mehrere Tage Wachleute bei ihren Streifengängen durch die Berliner U-und S-Bahn begleitet. Des Weiteren habe ich leitfadengestützte Experteninterviews mit Wachleuten und Schutzpolizisten ebendort geführt. Auf diesen beiden Quellen baut der folgende Abschnitt auf. Ich frage also: Welche Funktion von Wachleuten im ÖPNV könnte die Annahme rechtfertigen, dass sie einen Beitrag zur Verbrechensbekämpfung oder Kriminalitätskontrolle leisten?

Bei ihrer gänzlich unspektakulären Tätigkeit - die Wachleute fahren in der Regel acht Stunden lang mit dem Zug - kommen sie mit Tausenden von Passagieren in Kontakt. Entweder sind sie - vor allem nachts eine von den Verkehrsbetrieben immer häufiger angeordnete Maßnahme im Rahmen einer Sicherheitsstrategie - einem bestimmten Zugumlauf zugeteilt, den sie nur bei besonderen Vorkommnissen verlassen dürfen. 
Häufig müssen sie sich sogar in einem bestimmten Abteil aufhalten, vorzugsweise dem vorderen, um dort den Fahrgästen als Ansprechpartner zur Verfügung zu stehen. Dann fahren sie von Endstation zu Endstation und wieder zurück. Die Langeweile, die sich hier einstellt, kennzeichnet ihren Beruf. Erschwerend kommt hinzu, dass sie sich nicht setzen dürfen, sondern stehen müssen. Vor allem ältere Mitarbeiter bewegen sich deshalb nur sehr langsam vorwärts, wenn sie gehen, und wirken geradezu apathisch. Allein manche der Jüngeren sind eifrig, engagiert und unerschrocken bemüht, ihren Auftrag aktiv auszuführen.

Wenn in den Abendstunden und nachts die Zahl der Fahrgäste abnimmt, werden die nicht mehr benötigten Züge in den Kehren abgestellt bis zum nächsten Einsatz am nächsten Morgen. Vor allem während der Spätschicht werden Wachleute zu sogenannten Kehrfahrten beordert. Dann müssen sie einen oder mehrere Züge bis zum nächsten Morgen bewachen. In regelmäßigen Abständen gehen sie um die Züge herum und prüfen sie auf Bemalung und Beschädigung. An einem elektronischen Lesekontrollgerät belegen sie, dass sie ihren Rundgang gemacht haben. Kehrbewachung ist wichtig für die Verkehrsbetriebe, weil man bemüht ist, keine bemalten oder beschädigten Züge in den Tagesbetrieb zu nehmen; man will den Kunden ausschließlich saubere und ordentliche Verkehrsmittel bieten. Für die Sicherheitsdienste ist die Verhinderung der genannten Schäden bares Geld wert, weil der Bewachungs(miss)erfolg in der Größe der besprühten Fläche gemessen werden kann. Unterstützt in ihrem Ziel werden die Verkehrsbetriebe durch die Kommunen, für die der Nahverkehr inzwischen nicht mehr das Stiefkind der Lokalpolitik ist, sondern als Visitenkarte der Stadt angesehen wird und entsprechend gepflegt werden mus.

Die Kehranlagen vor allem, aber nicht nur sie, sind durch die Neubewertung des ÖPNV zu einem heiß umkämpften Gelände geworden, auf dem sich ein Kleinkrieg zwischen jugendlichen Sprayern auf der einen und der Polizei und den Wachleuten auf der anderen Seite abspielt. Hier prallen unterschiedliche Nutzungsansprüche ohne Aussicht auf Vermittlung aufeinander. Gegen die Vorstellung der Städte vom ÖPNV als ökonomisch effizient zu nutzendes Produktionsmittel im globalen Wettbewerb der Städte steht das Konzept der Sprayer: »Graffiti heißt >ich war hier < und ist Protest gegen das stillschweigende eintönige Einverständnis, dass die Stadt, die Öffentlichkeit irgendjemandem gehört.«(Wolf 200I) In diesem Konflikt stehen die Wachleute zuweilen in der ersten Reihe. Ein Wachmann bezeichnete im Interview die Graffiti-Szene als »eine sehr kriminelle Szene«, gut organisiert und ausgerüstet, strategisch denkend und gewaltbereit. Aber nicht nur wenn Wachleute Sprayer ertappen und stellen wollen - Fluchtversuche versucht man zu verhindern -, kommt es in den Kehranlagen zu gewaltsamen Auseinandersetzungen. Mein Interviewpartner berichtete auch von zwei Überfällen auf Kollegen, bei denen es den Angreifern darum ging, den Wachleuten ihre Jacken und Funkgeräte zu entwenden. Schläge müssen aber auch die Sprayer einstecken, wenngleich weniger von den Wachleuten, die man ohnehin nicht besonders ernst nimmt, sondern vor allem von zivilen Kriminalbeamten und BGS-Beamten. Der Wachschutz hingegen wird als »vollkommen harmlos « charakterisiert (Wolf 200I).

Der Krieg in den Kehren findet weitgehend im Verborgenen statt. Die Durchsetzung des Generalauftrags, die Betriebsordnung des Verkehrsträgers durchzusetzen bzw. wiederherzustellen, geschieht dagegen in aller Öffentlichkeit. Ordnung ist die Abwesenheit von deviantem Verhalten. »Das bloße Verweilen im Bahnhofsbereich (einschließlich der Zugangsbauwerke) ist nicht gestattet, « heißt es ultimativ in den Beförderungsbestimmungen des Berliner ÖPNV. Und: Hunde »sind kurz an der Leine zu führen. «In diesem Zusammenhang hatte ich während meiner Beobachtung ein bemerkenswertes Erlebnis. Bei der Diensteinweisung erhält das Team die Infor- 
mation, dass an einem Bahnhof Fahrgäste angebettelt würden. Die beiden fahren hin; ein paar Meter vor dem Ausgang des Bahnhofsgebäudes befinden sich zwei jugendliche Aussteiger mit ihrem Hund. Einer der beiden Wachleute fordert sie barsch auf, sich zu entfernen. Einer der Aussteiger ist sichtlich erregt und verzweifelt. Er deutet auf eine unsichtbare Linie am Boden, hinter die ein anderes Team der Sicherheitsfirma die beiden verbannt habe. Er will deutlich machen, dass er bereits einen Kompromiss eingegangen und Abmachungen auch von den Wachleuten einzuhalten seien. Die Wachleute zeigen sich davon unbeeindruckt, beharren auf ihrer Forderung und ziehen wieder davon, ohne später zu prüfen, ob die beiden Jugendlichen der Anweisung folgen.

Die Wachleute erreichen ihre Ziele, indem sie unerwünschte Personen immer wieder ansprechen, bis diesen als bessere Alternative zu den ständigen Störungen erscheint, sich dem Wirkungskreis der Uniformierten fernzuhalten - also zu gehorchen. Die beiden Aussteiger im Beispiel befanden sich kurz vor der »Kapitulation«: Die Verzweiflung des einen war die zur Erkenntnis sich verdichtende Ahnung, dass die Wachleute ihn nicht in Ruhe lassen würden, dass auf den einen bald der nächste folgen würde und dass als unter diesen Umständen »rationale A Alternative nur der Rückzug in Frage käme. So entfaltet der Einsatz der Wachleute im ÖPNV seine Wirkung: Man spricht an, bittet, fordert auf, droht. Der Eingriff in das Leben der Betroffenen ist von bloß geringer Intensität, das alleinige Einsatzmittel ist die Sprache. Über weitere Einflussfaktoren lassen sich bloß Vermutungen anstellen: Möglicherweise haben die Punker die Erfahrung gemacht, dass die Wachleute bei einem Konflikt auf die Rückendeckung durch die Polizei vertrauen können und die Staatsmacht ihnen wie ein Schatten folgt. Sicher ist nur, dass die Wachleute mit ihren beschränkten Mitteln unerwünschte Personen dauerhaft vertreiben können. Allein mit Prävention oder Verbrechensbekämpfung haben diese Maßnahmen nichts zu tun.

Wenn Wachleute Fahrkarten kontrollieren, muss dies in der Regel anlassbezogen geschehen, das heißt es muss ein normabweichendes Verhalten des Kontrollierten vorliegen. Das kann »verdächtiges « Verhalten sein, wenn ein Fahrgast von den Wachleuten nicht gesehen werden will oder plötzlich das Weite sucht. Aber auch wer die Füße auf den Sitz legt, unbefugterweise die Bahngleise überquert oder auf nichts zu warten scheint, muss mit einer anlassbezogenen Kontrolle rechnen; die Anlässe sind vielfältig. Wer keinen gültigen Fahrschein vorweisen kann, kann ein erhöhtes Beförderungsentgelt (EBE) von 60 DM entrichten und seiner Wege ziehen. Wer das nicht kann oder will, muss dem Verkehrsträger seine Identität offenbaren, indem er den Wachleuten glaubhaft seine Meldeadresse nennt oder »freiwillig « einen Ausweis vorweist. Selbstverständlich sind die Wachschützer nicht berechtigt, Identitätsfeststellungen vorzunehmen, aber sie können die Schwarzfahrer um den Ausweis »bitten« oder sie in dem Glauben lassen, sie seien berechtigt. Im Zweifelsfall wird ohnehin die Polizei eingeschaltet, die sodann die Prüfung vornimmt.

Die Berliner Verkehrsbetriebe (BVG) schicken zusätzlich zu einer Privatfirma eigenes Personal mit demselben Auftrag in den Untergrund, das von Beamten des I98 I per Senatsbeschluss ins Leben gerufene »Einsatzkommando BVG« der Berliner Polizei begleitet wird. Auf diese Weise ergibt sich eine praktische Arbeitsteilung, weil die Staatsmacht bei Konflikten, die sich aus Hausrechtsmaßnahmen ergeben, nicht erst umständlich gerufen werden muss, sondern gleichzeitig tätig werden kann. Damit ergibt sich bei Kontrollen durch eine gemischte Streife eine deutlich höhere Intensität. Der BVG-Mitarbeiter prüft den Fahrschein bzw. leitet die für die Anzeige notwendigen Formalitäten ein, während der Beamte eine Identitätsfeststellung - mit der Gefahr der Fesselung und Durchsuchung, falls der Kontrollierte angibt, keine 
Papiere mit sich zu führen - vornimmt. Bei Musikern wird routinemäßig vom BVG-

Mitarbeiter die Spielgenehmigung und vom Beamten der Pass geprüft. Aber diese gemischten Streifen sind eine Berliner Besonderheit. Üblicherweise werden die kooperationsunwilligen Schwarzfahrer so lange festgehalten, bis eine von der Leitstelle alarmierte Streife von BGS oder Landespolizei eintrifft.

In den Fahrscheinkontrollen erfüllen die Privaten tatsächlich repressive Aufgaben, denn »Schwarzfahren« ist eine Straftat ( $\$ 265$ a StGB [Leistungserschleichung]), und ein EBE zu verlangen, stellt die einzig mögliche Vertragsstrafe dar, die die Verkehrsbetriebe in Gestalt ihrer Beauftragten verhängen dürfen. Die Wachleute bekämpfen in diesem Sinne also das Verbrechen, indem sie Straftaten ermitteln und ahnden. Und wenn sich der Schwarzfahrer nicht kooperativ zeigt, dann können die Wachleute sogar dazu beitragen, weitere Straftaten aufzudecken, wenn nämlich die Polizei hinzugerufen und eine weitergehende Kontrolle vorgenommen wird. Aber sinnvoll kann von Verbrechensbekämpfung dennoch nicht die Rede sein, weil Schwarzfahren schwerlich als Verbrechen angesehen werden kann (vgl. Martin i995). Viel eher produzieren die Wachleute einen erhöhten Arbeitsanfall für die Polizei und weitere Straftaten, wenn sich etwa ein Ertappter unglücklich aus einer peinlichen Lage befreien will.

Von der Notwendigkeit, im ÖPNV besondere Anstrengungen zur Verbrechensbekämpfung zu unternehmen, kann nicht die Rede sein, weil dort nach übereinstimmender Auffassung das Straftatenaufkommen so gering ist wie nirgends sonst (vgl. Senat von Berlin 1999: 3). Erklärtermaßen richtet sich der Einsatz der Privaten entsprechend nicht gegen (potenzielle) Straftäter, sondern ist Teil eines umfassenden Konzepts, das das subjektive Sicherheitsgefühl erhöhen will. Unsicher fühlt man sich jedoch nicht, weil man droht, Opfer einer Straftat zu werden. Vielmehr entsteht Unsicherheit aus "Situationen der Hilflosigkeit und des Misstrauens, aus Gefühlen von Ohnmacht, des Vernachlässigt- und Alleingelassen-Werdens, aus fehlenden Möglichkeiten - oder zumindest des Wissens um solche -, >tätigく sein zu können und durch eigene Handlungen Einfluss zu nehmen, also eine (relative) Kontrolle der eigenen Umwelt auszuüben.«(Karazman-Morawetz 1996: 36) Folglich versucht man, den ÖPNV »sicher « zu machen, indem man Sicherheit mit Sauberkeit und anderen Wohlfühlfaktoren gleichsetzt, und legt dabei mehr oder weniger bewusst die Broken-windows-Theorie (vgl. Wilson/Kelling 1996) zugrunde, die dem bürgerlichen Ressentiment - Sicherheit als Sauberkeit - eine wissenschaftliche Weihe verleihen soll. Die Sicherheit der ÖPNV-Nutzer als Kunden - hergestellt durch gepflegte Züge, helle Tunnels, Fernsehen in der U-Bahn und die Abwesenheit von Randgruppen - geht allerdings zu Lasten der Nutzer, die zum Sicherheitsrisiko erklärt und verwaltungstechnisch bearbeitet werden.

Vom zusätzlichen Arbeitsanfall durch Bagatelldelikte und aus sich aus Konflikten bei der Feststellung derselben ergebenden Straftaten und Ordnungswidrigkeiten abgesehen, ist der Einsatz der Privaten für die Polizei insofern vorteilhaft, als das Aufspüren von abweichendem - also verdächtigem - Verhalten ein geeigneter Anlass zur Personenkontrolle ist. Die Privaten treiben mit der Aufklärung der Schwarzfahrerdelinquenz die Aufklärungsrate insgesamt in die Höhe und verhelfen der Polizei zu dem einen oder anderen Fahndungserfolg.

\section{Polizeiliche Videoüberwachung in Leipzig}

Sicherheitsproduktion ist zu einem Gutteil tatsächlich Produktion von materiellen Gütern und nicht nur Dienstleistung wie im vorangegangenen Kapitel dargestellt. Die Industrie entwickelt Produkte, die sie dem Staat zur Verfügung stellt. Der Privat- 
sektor beteiligt sich also indirekt auch an hoheitlichen Maßnahmen, denn er schafft mit seinen Innovationen erst die Voraussetzung für verschiedene Strategien der Polizei. Da der Stand der Technik direkten Einfluss auf staatliches Handeln hat, kann von einer Abhängigkeit der Polizei von der Industrie gesprochen werden. Diese wird sich in Zukunft noch vergrößern, weil die »Sicherheitstechnik mit ihren großen Möglichkeiten... [als ein] ganz wichtiger Bestandteil der Prävention« verstanden wird (Schily 2000: 3).

Videoüberwachung ist nicht neu, bloß hat sich erst seit wenigen Jahren die Technik so weit entwickelt und das kollektive Bewußtsein sich so weit verändert, dass ihr Einsatz jetzt attraktiv wird. Die Kameras werden kleiner und leistungsfähiger, und es können durch Digitalisierung größere Datenmengen über weitere Entfernungen transportiert und platzsparender gespeichert werden als je zuvor. Neben den nunmehr primitiv anmutenden Fixkameras zur Überwachung eines Tores, eines Zauns und dergleichen existieren inzwischen sog. Dome-Kameras; sie haben einen »Schwenkbereich von $360^{\circ}$ (kein Anschlag), schnelle Positionierungszeiten ( $>60^{\circ} /$ Sek.) sowie eine Autofokusfunktionalität.«(ZVEI 2000: 29) Manche schalten automatisch bei Dämmerung auf Infrarot-Beleuchtung um, man kann »privacy-zones « definieren, und ganz innovative Systeme können sogar »verdächtiges Verhalten « erkennen und Alarm schlagen; andere gleichen die aufgezeichneten Gesichter mit den in einer digitalen Kartei gespeicherten Bildern ab (FAZ I. März 200I). Die Bilder werden bis zu einer Entfernung von 70 Kilometern über Monomode-Glasfaserkabel oder gleich über ISDNFernmeldeleitungen übertragen (ZVEI 2000: 3I). Wie die zahlreichen Webcams beweisen, kann man mit einer Kamera in Berlin aufgenommene Bilder problemlos in Echtzeit auch in Australien verfolgen und auswerten. Der Ort der Aufnahme und der Ort des Empfangs sind jedenfalls nicht notwendig derselbe, sondern vollständig entkoppelt. »Die neueste Generation der Kuppelkameras von Sensomatic, " heißt es in einer Fachzeitschrift über das Firmenprodukt, »der Deltadome II, bietet Hochleistungstechnik, wie Zielanfahrt innerhalb von I,6 Sekunden, I76-fach Zoom, Autofokus und Blendenautomatik, komfortable Bedienung durch Joystick oder Trackball, sowie digitales Signalprozessing (DSP 3) zur Bildoptimierung. (CD-Sicherheitsmanagement 4/2000: I67)

Die Erkenntnis, dass die private Wirtschaft der beste Kunde der Sicherheitsindustrie ist, gilt für das Produkt Sicherheitstechnik gleichermaßen. Während der polizeiliche Einsatz der Videoüberwachung gleichsam noch in den Kinderschuhen steckt und erst Anfang der neunziger Jahre in Deutschland die ersten Versuche angelaufen sind, wird auf Privatgrund schon lange - an Geldautomaten, Ladenpassagen etc. - aufgezeichnet, wo den Unternehmen materieller Schaden droht. Der Staat hat sich bislang auf die zusätzliche Sicherung von Botschaften, Ministerien und Flughäfen beschränkt. Die Videoüberwachung wurde als geeignetes Präventionsmittel angesehen, aber nicht eines der Verbrechensbekämpfung.

Doch inzwischen hat die Polizei die Videoüberwachung als geeignetes Mittel für sich entdeckt und beginnt, sie systematisch anzuwenden. Als Dammbruch kann der Aufbau eines Systems 1996 durch die Leipziger Polizei gelten, die in einem auf vier Wochen angelegten Pilotprojekt auf dem Dach eines Hauses in der Nähe des Hauptbahnhofs eine schwenk- und zoomfähige Kamera installiert hatte; ein Hinweisschild wurde aufgestellt. Man hatte in dem ins Visier genommenen Bereich ein erhöhtes Straftatenaufkommen durch Btm- und Nachfolgedelikte registriert. Von Stasi-Methoden hat man sich jedoch distanziert. Nach einer Pause von einem halben Jahr wurde ein zweites Pilotprojekt für sechs Monate am selben Standort bewilligt. Weil auch dieses zweite Pilotprojekt als erfolgreich bewertet wurde, wurde eine unbefristete Fortsetzung der Videoüberwachung an diesem Standort beschlossen. 
zu der Bewertung, dass »die Videoüberwachung ausgewählter Kriminalitätsbrennpunkte als Bestandteil eines Maßnahmenkomplexes« ein »geeignetes und angemessenes polizeiliches Mittel zur Kriminalitätsbekämpfung « sei (Müller ı998: I I6).

Aufgrund dieser positiven Bewertung wurde die Kameraüberwachung in Leipzig ausgedehnt. Im November 1999 wurde am Connewitzer Kreuz eine Kamera montiert, weil es sich hier »seit der Wende um einen besonderen Gefahren- und Gefährdungsraum « handele. »Dort kam es in der Vergangenheit immer wieder zu Auseinandersetzungen gewalttätiger Jugendlicher mit der Polizei.« (Müller 2000: 288) Während der fünfmonatigen Überwachungszeit kamen der Polizei keine Straftaten mehr zur Kenntnis. Die Bevölkerung wehrte sich inzwischen mit Demonstrationen und Diskussionsveranstaltungen gegen die Dauerbeobachtung. Am i 9. April 2000 wurde die Kamera wieder außer Betrieb genommen. Ob wegen der Proteste oder weil die Polizei den Einsatz als abgeschlossene Maßnahme einstufte, lässt sich aus den Aufsätzen Müllers nicht erschließen. Noch am selben Tag wurde mit der Überwachung von zwei weiteren Bereichen - am Martin-Luther-Ring und am Rossplatz - begonnen. Alle nunmehr drei überwachten Bereiche befinden sich an Straßen, die ringförmig um die Leipziger Innenstadt führen.

Die polizeiliche Videoüberwachung wurde zu einer dauerhaften Einrichtung an Orten, die die Polizei als Kriminalitätsschwerpunkte identifiziert hat. Auto- und Taschendiebstahl, Raub, Erpressung, Körperverletzung und Drogendelikte spielten nach Angaben der Polizei in diesen Bereichen die maßgebliche Rolle. Durch die Videoüberwachung habe man die Häufigkeit dieser Delikte senken und eine »Stabilisierung auf verhältnismäßig niedrigem Niveau « herbeiführen können. Sie habe sich als ein geeignetes Instrument der Prävention erwiesen; man habe 23 Täter mit ihrer Hilfe stellen können. Kriminalität sei nicht in andere Stadtteile verdrängt worden. Durch den Überblick, den die Kameras gewähren, könne die Polizei die Lage vor Ort jetzt besser einschätzen. Lediglich die Btm-Szene formiere sich seit Beginn der Überwachung an anderen Orten neu (Müller 2000: 29I).

Aus polizeilicher Sicht hat sich der Einsatz der Videokameras also angeblich gelohnt. Die Fallzahlen gingen in den überwachten Bereichen zurück, insofern scheint sich der Einsatz der Kameras als probates Mittel wenn schon nicht der Repression, so doch der Prävention zu empfehlen. Allerdings präsentieren die Zahlen der Polizei ein lediglich sehr eingeschränktes Bild der Wirklichkeit und sind deshalb eine unzureichende Basis zur Bewertung von sozialen Prozessen wie Kriminalität. Was als vermeintlich objektive Größe in der PKS publiziert wird, ist das Ergebnis eines komplexen Konstruktionsprozesses, in dessen Verlauf etwa die Motive einer Anzeige bei der Polizei keinen Eingang in das durch die Filtermöglichkeit der aufnehmenden Beamten einerseits und das standardisierte Verfahren anderseits geprägte Verfahren der Einleitung eines Strafverfahrens finden (vgl. Busch/Werkentin I992). Insofern sind die von Müller präsentierten Daten lediglich Ausdruck der Wahrnehmung der Polizei und Grundlage für behördenimmanente Problemlösungsstrategien. Prozesse, die außerhalb dieser Logik stattfinden, können nicht in die Urteilsfindung einflieBen.

Aber aus den Ausführungen Müllers wird zudem deutlich, dass die Videoüberwachung auch aus polizeilicher Sicht als verfehlt hätte eingestuft werden müssen. Denn Ausgangspunkt der Überlegungen war die Herausforderung der Polizei durch die am Hauptbahnhof etablierte Btm-Szene und die mit dem Drogenhandel und -konsum einhergehende Beschaffungskriminalität. Anwohner und Geschäftsleute waren verunsichert. Um die Szene aufzulösen, hat man, berichtet Müller, zeitweilig den Kontrolldruck erhöht und die Bevölkerung auf die Gefahr des Kfz-Diebstahls an diesem 
Ort hingewiesen, während die Stadt gleichzeitig eine Allgemeinverfügung gegen die Drogenhändler und -konsumenten erlassen hat (vgl. Müller I 997: 77). Gefruchtet haben die Maßnahmen jedoch nichts, wie Müller selbst zugibt. Die Szene ist lediglich in die nicht-überwachten Bereiche ausgewichen (Müller 2000: 29I), wo sie wiederum neue Polizeikräfte binde (Müller ı998: I I6).

So bleiben bei aller Freude über gesunkene Deliktzahlen im überwachten Bereich zwei ungeklärte, aber durchaus entscheidende Fragen, die am Sinn der Videoüberwachung zweifeln lassen: In welchem Zusammenhang steht der allgemeine Rückgang des Kfz-Diebstahls in Leipzig-Mitte zu dem Rückgang im Überwachungsbereich (vgl. Müller I 998: i I 5) ? Und wie und wo beschaffen sich die Süchtigen, nachdem sie vom Platz vor dem Hauptbahnhof verdrängt wurden, jetzt das Geld zum Kauf von illegalen Drogen, das sie zuvor dort erbeutet haben? Durch die Polizeimaßnahme sind sie zwar aus dem Gesichtskreis der Polizei verschwunden, unsichtbar gemacht, aber sie sind zweifelsohne weiterhin vorhanden und weiterhin süchtig. Die Beurteilung der Videoüberwachung durch die Polizei macht vor allem deutlich, dass die Interpretation der Ergebnisse der Überwachung eher von Wunschdenken als von Bezug zur Wirklichkeit geprägt ist.

Die Videoüberwachung ist aber nicht nur wirkungslos in Blick auf die selbst gesteckten Ziele. Vielmehr hat es den Anschein, dass sie - unausgesprochen, aber angesichts des mangelhaften Erfolgs um so deutlicher zu erkennen - als Disziplinierungsmittel für bestimmte Bevölkerungsgruppen eingesetzt wird und sich deshalb in erster Linie diskriminierend auswirkt. Das erste Pilotprojekt richtet sich vorwiegend gegen »Ausländer aus Nordafrika, dem südosteuropäischen und vorderasiatischen Raum «, wie es im Einsatzbefehl an die Leipziger Beamten heißt (vgl. Müller I997: 78 f.). Während hier rassistisch gefärbte Vorurteile als Kriminalitätsbekämpfung getarnt werden, dient die Kamera am Connewitzer Kreuz zur Disziplinierung der vorwiegend aus Jugendlichen bestehenden autonomen Szene. Damit erweist sich der Technikeinsatz als Verlängerung des ohnehin verfehlten Ziels, soziale Probleme mit polizeilichen Methoden lösen zu wollen. Ruhe und Ordnung, die nicht durch Sucht und Aufmüpfigkeit gestört wird, wird in die polizeilich handhabbare Kategorie der »Kriminalität « gegossen. Paradoxerweise besteht die Wirkung der Beobachtung in der Unsichtbarmachung. Dass dadurch die Probleme nicht verschwinden, sondern im Gegenteil durch Verschärfung der »Unsichtbarkeitsfalle« ein neues Gewaltpotential geschaffen wird (vgl. Schroer 2000: 447), versteht sich fast von selbst.

Der Fall Leipzig demonstriert eine weitere Gefahr, die sich aus dem Einsatz neuer Technologien ergibt. Innovationen haben die Eigenschaft, eine Eigendynamik zu entwickeln, die, vor allem in der Hand der mit dem Monopol legitimer physischer Gewaltsamkeit ausgestatteten Polizei, nur schwer zu kontrollieren ist. Man gewöhnt sich an das neue Mittel; die Hemmschwelle, es einzusetzen, sinkt. Der Einsatz löst sich von dem zuvor selbst gesetzten Rahmen. Der Zwang, den Einsatz zu legitimieren, schwindet, weil sich bei den Unterworfenen ein Gewöhnungseffekt bemerkbar gemacht hat. Der Einsatz am Hauptbahnhof erfüllt nicht den eingangs verfolgten Zweck, wird aber dennoch fortgesetzt. Die Hemmschwelle für einen weiteren Einsatz ist dennoch gesunken. Es ist, als sei ein Damm gebrochen. Am Hauptbahnhof wurden zu Beginn der Beobachtung $70 \mathrm{Kfz}$-Diebstähle, I 3 Taschendiebstähle, 8 Raubtaten, 5 gemeldete Körperverletzungsdelikte und I 8 Btm-Straftaten in einem Monat gemeldet. Für den Einsatz am Connewitzer Kreuz mussten 7 Taschendiebstahlsdelikte, $69 \mathrm{Kfz}$-Diebstähle, 7 Raubtaten, 38 Körperverletzungsdelikte, $3 \mathrm{Btm}$ Delikte und I 2 Fälle von Sachbeschädigung in den zebn Monaten vor der Überwachung als Legitimation herhalten. Von dem nicht vergleichbaren Delikt der Sachbeschädigung abgesehen, lag die Häufigkeit also bei circa ı०\% oder noch darunter. 
Wenn vom Connewitzer Kreuz als einem »besonderen Gefahren- und Gefährdungs-

raum « die Rede ist, kann die Kriminalitätsrate nicht gemeint sein, denn die ist - im Vergleich zum Hauptbahnhof - eher niedrig. Es ist kein Wunder, dass deshalb das immer wieder strapazierte »subjektive Sicherheitsgefühl der Bevölkerung in diesem besonderen >Angstraum« (Müller 2000: 288) als Legitimation herhalten muss, weil sogar die polizeispezifischen Argumente ausgegangen sind.

Doch nicht genug, dass Videoüberwachung in Leipzig nicht nur keine nachhaltigen Erfolge bei der Kriminalitätsbekämpfung zeitigt und de facto als Maßnahme zur Kontrolle der gefährlichen Klassen eingesetzt wird - sie ist eine echte Gefahr für die Bürgerrechte auch derer, denen Schutz durch die Maßnahme verheißen wird. Kameras disziplinieren das Verhalten in jedem Fall, indem sie einen subtilen Druck ausüben, nicht aufzufallen. Wer sich beobachtet weiß, steht unter einem permanenten Stress, der nicht nur psychische, sondern auch politische Auswirkungen haben kann. Den Zusammenhang von persönlicher Integrität und politischer Freiheit hat das Bundesverfassungsgericht im Volkszählungsurteil von 1983 in schwer zu überbietender und deshalb noch einmal ausführlich zu zitierender Weise deutlich gemacht: »Wer unsicher ist, ob abweichende Verhaltensweisen jederzeit notiert und als Information dauerhaft gespeichert, verwendet oder weitergegeben werden, wird versuchen, nicht durch solche Verhaltensweisen aufzufallen. Wer damit rechnet, dass etwa die Teilnahme an einer Versammlung oder einer Bürgerinitiative behördlich registriert wird und dass ihm dadurch Risiken entstehen können, wird möglicherweise auf eine Ausübung seiner entsprechenden Grundrechte (Art. 8, 9 GG) verzichten. Dies würde nicht nur die individuellen Entfaltungschancen des Einzelnen beeinträchtigen, sondern auch das Gemeinwohl, weil Selbstbestimmung eine elementare Funktionsbedingung eines auf Handlungs- und Mitwirkungsfähigkeit seiner Bürger begründeten freiheitlichen demokratischen Gemeinwesens ist.«(BVerfGE 65, 43)

\section{Werkschutz und Privatjustiz}

Beim Werkschutz handelt es sich um eine klassische Form der Herstellung von Sicherheit durch nicht-staatliche Stellen. Im Grunde ist der Betrieb das klassische Betätigungsfeld für private Sicherheitsdienste. Was ihn heute von dem Werkschutz vor hundert Jahren unterscheidet, ist im Wesentlichen, dass die Dienstleistung immer häufiger nicht von eigenen Kräften des Betriebs erbracht wird, sondern Fremdfirmen beauftragt werden (»Outsourcing«). Die Schwächung sozialer Kontrolle im Verlauf der industriellen Revolution erforderte neue Formen des Eigentumsschutzes, zumal in größeren Betrieben. Nicht von ungefähr entstanden die ersten Fabrikdiebstahlvereine, die Vorläufer des Werkschutzes, im Ruhrgebiet. In einer Lesart etablierte sich der Werkschutz als private Form der Gefahrenabwehr nicht nur als Reaktion auf die zunehmende Betriebsgröße, sondern auch als Reaktion auf das Unvermögen der Polizei, die Kriminalität in den Betrieben selbst zu bekämpfen, weil die Fälle zu komplex und zu zahlreich waren. Da die Kriminalität lediglich innerhalb des Betriebs stattgefunden habe, die Bekämpfung durch den Werkschutz zugleich jedoch der Allgemeinheit diene, sei der Werkschutz durchaus im Einvernehmen mit der Polizei entstanden (Bauer 1985: I 2 f.). Diese Interpretation ist insofern zulässig, als sich die privaten Sicherheitsdienste tatsächlich in Reaktion auf die Umorganisation staatlicher Formen der Sicherheitsgewährleistung - Verstaatlichung der städtischen Schutzmannschaften I 892/95 - entfalteten.

Allerdings sollte kein Missverständnis über die rechtliche Stellung des Werkschutzes und über sein Verhältnis zur Polizei aufkommen. Der Werkschutz hat keine repressiven Befugnisse, sondern ist »ein Organ der Gefahrenabwehr des gesamten Objekts 
mit ausschließlich sachbezogenen Aufgaben«, wie es im zweiten Leitsatz für den Objektschutzdienst (also den Werkschutz) heißt. Personen, Sachen und Ereignisse bzw. Zustände können Gefahrenquellen für die - weit verstandene - Betriebssicherheit sein und müssen deshalb kontrolliert werden (vgl. Kötter 1995: 318). Der Generalauftrag lautet dementsprechend: »Gefahren und Schäden verhindern. Ordnung und Sicherheit gewährleisten.«(Ehses 1995: 445) Verbrechensbekämpfung gehört also nicht zu den Aufgaben des Werkschutzes, sondern bleibt der Polizei vorbehalten. Prävention, Gefahrenabwehr, Eigensicherung - das ist die Domäne des Werkschutzes.

Seine Aufgabe erfüllt der Werkschutz vorrangig durch zahlreiche, permanente Kontrollen aller Personen, Sachen und Zustände, die in irgendeiner Weise die Belange des Betriebs betreffen. An den Pforten werden die Dienstausweise der Mitarbeiter geprüft, an Besucher werden Besucherausweise ausgestellt. Dienstfahrzeuge werden auf ihre Verkehrstauglichkeit untersucht und die Lieferungen von Firmenkunden auf Gefährdungspotential für den Betrieb. Personen- und Fahrzeugkontrollen sollen wertneutral durchgeführt werden und also jedermann treffen können. Es werden zumeist Zufallsstichproben durchgeführt; ein konkreter Verdacht muss nicht vorliegen, wenn bspw. jede zehnte Person oder jedes zwanzigste Fahrzeug kontrolliert wird. Solche präventiven Kontrollen erstrecken sich über das gesamte Werksgelände und können alle Personen und Fahrzeuge jederzeit treffen. Das Risiko einer Fahrzeugkontrolle - wie das der Personenkontrolle - »darf für Fahrer und Begleitperson nicht kalkulierbar sein. (Ehses I 995: 452) Das Charakteristische für die Werkspolizei ist also, dass sie angehalten ist, jedermann auf dem Werksgelände für verdächtig zu halten und ein dieser Prämisse entsprechendes Kontrollkonzept zu entwickeln. Dadurch schwindet die Grenze zwischen präventiver und repressiver Tätigkeit bis zur Unkenntlichkeit, so dass Bauer von diesen »Repressivmaßnahmen« als »Präventivmaßnahme[n] (mit Repressivcharakter)« spricht (Bauer I98 5: I 23).

Ebenfalls als präventive Maßnahme rubriziert werden die Kontrollgänge der Werkschutzmitarbeiter, bei denen sämtliche sicherheitsrelevanten Bereiche bestreift und auf störungsfreien Betrieb bzw. Unversehrtheit geprüft werden. Sie achten auf Einhaltung der Unfallverhütungsvorschriften, kontrollieren die Sicherheitsvorkehrungen in störempfindlichen Bereichen ebenso wie die Zutrittsberechtigung etwa zur EDV-Zentrale. Türen müssen geschlossen werden, Fluchtwege freigehalten und sonstige kleineren und größeren Versäumnisse des Personals korrigiert werden. Sie überprüfen abgestellte Fahrzeuge und untersuchen Gebäude und Anlagen auf mögliche Schäden. Der Umzäunung und abgelegenen Werksbereichen widmen sie ebenfalls ihre Aufmerksamkeit. Aber ihr Blick reicht auch über den Zaun hinaus in das nähere Umfeld des Werks, das ebenfalls in Augenschein genommen wird (Ehses I 995: 456). Der Werkschutz unterwirft das Umfeld seinen Kontrollansprüchen und dehnt sie im Bedarfsfalle sogar noch weiter aus, wenn beispielsweise des Diebstahls verdächtigte Personen observiert werden oder die korrekte Leistungserbringung einer Vertragsfirma (bspw. Müllentsorgung) kontrolliert wird (vgl. ebd.: 460).

Die Vorgehensweise des Werkschutzes bei der Täterermittlung ist deliktspezifisch unterschiedlich, es lassen sich jedoch einige Elemente herausfiltern: Zuerst wird derwenn vorhanden - Tatort abgesperrt, die Spuren gesichert, also Fingerabdrücke, Reifenspuren, Blut etc. sichergestellt und untersucht, Zeugen werden befragt. Wenn noch kein Tatverdächtiger ermittelt werden konnte, kann der Werkschutz Fallen aufbauen, die bei der Ergreifung etwa von Mehrfachdieben helfen sollen. Dann wird heimlich observiert, werden Klingeldrähte gespannt, Lichtschranken und Photoapparate montiert oder chemische Mittel gestreut (Riester I991: I04I09). Falls sich ein Verdächtiger herauskristallisiert hat, wird er unter Umständen 
unter Vortäuschung falscher Tatsachen ausgehorcht oder offen zur Befragung gebeten. Im Anschluss an die Ermittlungen gibt der Werkschutz die Akte an die Betriebsleitung weiter, die eine Betriebsbuße verhängen kann, die von der Ermahnung und dem Verweis über eine Geldbuße bis zur Versetzung und Entlassung reicht (Bauer I985: I 4I-I46) - entsprechend dem Opportunitätsprinzip des Arbeitgebers.

Solche Tätigkeiten weisen über die reine Gefahrenabwehr natürlich weit hinaus und werden deshalb auch von Bauer und Ehses »zur prekären Seite « objektschutzdienstlichen Vorgehens gerechnet. Repressive Tätigkeit, so heißt es, sei die Aufgabe von Strafverfolgungsbehörden, die sich bei ihren Ermittlungen an die eng gefassten Vorschriften der Strafprozessordnung halten müssen. Diese Bindung an das Strafprozessrecht, das das Vorgehen der Ermittlungsbehörden regelt und deshalb den bis zum Erweis seiner Schuld durch ein ordentliches Gericht als unschuldig geltenden Beschuldigten schützt, spielt bei den Ermittlungen des Werkschutzes praktisch keine Rolle. Das Strafverfolgungsmonopol wird zudem durch die Praxis der Unternehmen unterlaufen, ermittelte Straftaten hauptsächlich innerhalb des Betriebs zu sanktionieren und den Behörden in bloß I6\% der Fälle überhaupt zur Kenntnis zu geben (ebd.: I 52). Der Hinweis in den Lehrbüchern für den Werkschutz auf das Monopol des Staates ist insofern ohne praktische Relevanz. Das Monopol wird durch eine Analogiebildung, die die gesetzliche Form wahrt, unterlaufen und damit ein substaatliches Repressionssystem geschaffen, das offiziell auf der freiwilligen Mitarbeit von Beschuldigten - und der Nicht-Beschuldigten, die von permanenter verdachtsloser Kontrolle betroffen sind - beruht, in Wirklichkeit jedoch auf dem Abhängigkeitsverhältnis zwischen Arbeitgeber und Arbeitnehmer und damit auf einer subtilen, verinnerlichten Form von Zwang aufbaut.

Rechtsstaatliche Grundsätze spielen bei den Ermittlungen keine Rolle, weil die Materie gesetzlich nicht geregelt ist; handlungsleitend sind allein utilitaristische Abwägungen. Die StPO kann den Ermittlern »als Richtschnur (Britsch/Königseder I 997: 64) dienen, muss aber nicht. Der Verdächtige ist seinem Arbeitgeber auf Gedeih und Verderb ausgeliefert, weil die sogenannte Freiwilligkeit seiner Mitwirkung aus der Treuepflicht gegenüber seinem Arbeitgeber abgeleitet wird. »Da es einerseits wie erwähnt - keine konkreten Regelungen gibt, die den Rahmen und die Voraussetzungen für eine Vernehmung festlegen, fehlt es - logischerweise - auch an Vorschriften, die etwa unlautere Vernehmungsmethoden verbieten und Folgen androhen. Sicher macht sich auch der Werkschutz bei entsprechendem Verhalten nach $\$ \int 239$, 240 oder I 85 StGB strafbar, doch ist die Wahrscheinlichkeit, dass dieses Verhalten angezeigt wird, gering und hätte im übrigen keinen oder nur geringen Einfluss auf die Verwertbarkeit einer so erlangten Aussage.« (Bauer I985: I32) Im Lichte der Erkenntnis, dass rechtsstaatliche Grundsätze innerhalb des Betriebes keine handlungsleitende Funktion erfüllen, ist die Gefahr nicht zu unterschätzen, dass diese Methoden auch außerhalb des Firmengeländes angewendet werden, weil sie den Werkschutz-Mitarbeitern gleichsam in Fleisch und Blut übergegangen sind. Von Durchsuchungen von Privatwohnungen und Festnahmen außerhalb des Firmengeländes wird berichtet (vgl. ebd.: I 28).

Die Werkspolizei unterscheidet sich also in einem ganz wesentlichen Punkt von der öffentlichen Polizei, der bei der Beantwortung auf die eingangs aufgeworfene Frage nach der Integration der Privaten in die Verbrechensbekämpfung immer mitberücksichtigt werden muss. Die Kontrollen von Personen und Fahrzeugen, die jeden treffen können und sollen und nicht kalkulierbar sind, stellen die gesamte Belegschaft unter Generalverdacht. Sobald der Werkschutz Verdacht geschöpft hat, ist er in seinen Ermittlungsmethoden nicht beschränkt, sondern kann alle ihm zweckdienlich erscheinenden Maßnahmen ergreifen. Er ist nicht dem Allgemeinwohl verpflichtet, 
sondern dem partikularen Interesse seines Auftraggebers. Die als Freiwilligkeit bemäntelte Zustimmung der Firmenangehörigen zu den Ermittlungen des Werkschutzes beruht auf dem Abhängigkeitsverhältnis, das mit Arbeitsvertrag, Tarifverträgen, Betriebsvereinbarungen und Eigentumsrechten des Arbeitgebers abgesichert ist. Selbst wenn also das Recht durch den Werkschutz nur in den - wohlwollend unterstellt - wenigsten Fällen gebrochen wird, entsprechen die Methoden des Werkschutzes nicht denen der Polizei in einem freiheitlich-demokratischen Rechtsstaat.

So entsteht eine paradoxe Situation: Obwohl der Werkschutz eigentlich keine Eingriffsbefugnisse - also hoheitliche Befugnisse - hat, ist er in seinen Handlungsmöglichkeiten freier als die öffentliche Polizei, weil sein Instrumentarium wesentlich differenzierter als das der Polizei ist. Die Konflikte, mit denen der Werkschutz befasst ist, bewegen sich üblicherweise auf einem Niveau unterhalb der Schwelle zur körperlichen Gewalt. Sie sind so zahlreich und die Konfliktlösungskompetenz des Werkschutzes ist so umfassend, dass ihm dadurch eine nahezu schrankenlose Macht zukommt, die erst gebrochen würde durch die Notwendigkeit des Einsatzes körperlicher Gewalt. Seine Macht stützt sich auf ein dicht geknüpftes Netz von gesetzesgleichen privatrechtlichen Vereinbarungen, das als Tätigkeitsgrundlage völlig ausreichend ist. Verdeckte Ermittlungen, verdachtlose Kontrollen und andere unlauteren Methoden sind keine Gewalt im Sinne eines engen Gewaltbegriffs, auf den der der Polizei rekurriert, und also zulässig. Dass das Sicherheitsgewerbe keine über die bisherige Regelung hinausgehenden Befugnisse haben will, wie der Hauptgeschäftsführer des Bundesverbandes Deutscher Wach- und Sicherheitsunternehmen betont (Olschok 2000: 53), ist unter diesen mehr als günstigen Bedingungen nur verständlich. Das Gewerbe würde u U. in seiner Handlungsfreiheit beschränkt werden, wenn man ihm womöglich eine Art öffentliche Funktion zuweisen würde. Die Stellung des Werkschutzes außerhalb des öffentlichen Strafverfolgungssystem ist wesentlich vorteilhafter als eine Integration, weil er dann weiterhin nicht an hemmende rechtsstaatliche Grundsätze gebunden ist.

\section{Die Polizei als Werkschutz}

Inzwischen fransen die Grenzen zwischen dem Öffentlichen und dem Privaten jedoch aus, und von einer klaren Dichotomie von öffentlich und privat kann kaum mehr gesprochen werden (vgl. Jones/Newburn I 998). Damit geraten die Grundfesten des Verhältnisses von Bürgern zum Staat ins Wanken. Die Entwicklung des Rechtsstaats analog zu der Formierung der bürgerlichen Gesellschaft bedeutete die Beschränkung der Staatsgewalt und die Befreiung des wirtschaftlich aktiven Bürgertums von Bevormundung und Rechtsunsicherheit. Die Bedrohung bürgerlicher Freiheit, die sich nur in einem staatsfreien Bereich entfalten konnte, ging denknotwendig vom Staat aus, den man von Übergriffen auf diesen Bereich abhalten musste. Die Entwicklung der Massengesellschaft mit ihren spezifischen Produktions- und Vergesellschaftungsformen - Fabriken mit stadtähnlichen Ausmaßen, aber auch Einkaufspassagen, Vergnügungsparks, Wohnanlagen etc., also quasi-öffentliches privates Besitztum, das die klassische Trennung beider Sphären hinfällig werden lässt - war unmöglich zu antizipieren. Die Bedrohung bürgerlicher Freiheit geht deshalb inzwischen nicht mehr allein vom Staat aus, weil inzwischen großflächiger Privatgrund für den Publikumsverkehr geöffnet wurde, für den ein Sicherheitsdienst zuständig ist. Öffentliches Leben verlagert sich in den Herrschaftsbereich von Privatleuten (vgl. Shearing/Stenning 1983: $496 \mathrm{f}$.), weshalb die für die Abwehr staatlicher Gewalt konstitutive Unterscheidung zwischen der Sphäre des Öffentlichen und der des Privaten von Unternehmerseite aufgeweicht wird. 
Aber auch von staatlicher Seite wird diese Grenze überschritten. Es ist zu befürchten, dass der normative Unterschied zwischen der Polizei und dem Werkschutz an Bedeutung verliert, weil beide in Wechselbeziehung zueinander stehen und sich gleich einem »hydraulischen System « ergänzen: Die Polizei wird rechtsstaatliche Beschränkungen unter anderem durch die Kooperation mit dem an diese Beschränkungen nicht gebundenen Werkschutz umgehen wollen (vgl. Marx i987: i 85 f.). Erleichtert würde eine solche Zusammenarbeit durch personelle Überschneidungen, wenn leitende Polizeibeamte aus dem Dienst ausscheiden und eine Anstellung bei einem privaten Sicherheitsdienst erhalten. Aber auch wenn die Existenz eines solchen OldBoy-Networks unbestritten ist - viele Firmen brüsten sich geradezu mit den externen und »fachkundigen « Beratern und nehmen in ihre Beraterstäbe gerne auch Abgeordnete auf -, darf die davon ausgehende Gefahr für eine rechtstaatlich verfasste Polizei nicht übertrieben werden.

Entgegen der weit verbreiteten Auffassung, dass der Staat ein Monopol der Strafverfolgung hat, ist der Beitrag des privaten Sicherheitsgewerbes zur staatlichen Verbrechensbekämpfung dennoch nicht zu unterschätzen. Trotz formallogischer Trennung in einen öffentlichen und einen privaten Bereich ist das Monopol nicht so hermetisch wie angenommen. Wohl existiert eine theoretische Möglichkeit, sich gegen Maßnahmen des Werkschutzes zur Wehr zu setzen, aber sie ist ohne praktische Relevanz. Aber nicht nur der Auftraggeber, der Arbeitgeber, bevorzugt die informelle Konfliktregelung, auch die andere Seite - der Arbeitnehmer, der Kunde etc. - dürfte häufig daran interessiert sein, dass ein Konflikt nicht vor einem ordentlichen Gericht geklärt wird. Man könnte aus diesem Befund schließen, dass die Voraussetzungen für eine Integration der privaten Dienste in die Verbrechensbekämpfung günstig sind, weil man sich auf legaler und funktionaler Ebene ergänzen kann: Was der eine nicht darf und nicht kann, wird eben vom anderen erledigt.

Durch eine dergestalte Kooperation der Polizei mit dem Werkschutz würden jedoch die »machtbegrenzenden Verfassungsprinzipien« unterlaufen, mit denen die Arbeit der Polizei in einem Rechtsstaat notwendig behindert wird. Mit Roggan zu nennen sind hier erstens das Trennungsgebot zwischen der Ermittlungstätigkeit der Polizei zum Zwecke der Einleitung eines Strafverfahrens und der Nachrichtensammlung durch die Geheimdienste, um die Entwicklung eines allmächtigen Terrorapparates wie der Gestapo zu verhindern. Getrennt werden muss zweitens zwischen polizeirechtlichen und strafprozessualen Maßnahmen; die Polizei ist ein Organ der Gefahrenabwehr und darf sich deshalb nicht des Instrumentariums der Strafverfolgungsbehörden bedienen. Prävention und Repression sind grundsätzlich verschiedene Aufgaben verschiedener Behörden. Drittens leitet sich aus dem Ermittlungsgebot, das die Gleichbehandlung aller Bürger vor dem Recht im Sinn hat, ein Verbot zu ermitteln ab, wenn kein hinreichender Anfangsverdacht besteht. Jedermann hat als unschuldig und redlich zu gelten und nicht vom Staat belästigt zu werden, wenn sich kein Verdacht auf das Vorliegen einer Straftat ergibt. Überwachungsmaßnahmen dürfen nicht ins Blaue hinein geführt werden. Und wenn dann doch gegen ihn ermittelt wird, muss der Bürger, viertens, die gesetzlichen Grundlagen erfahren können, die sich durch Normenbestimmtheit und Normenklarheit auszeichnen sollen. Schließlich, das hängt eng mit diesem Prinzip zusammen, gilt die grundsätzliche Offenheit staatlichen Eingriffshandelns, damit sich der Betroffene gegen die Ermittlungen überhaupt erst wehren kann (Roggan 2000: 30-46). Die Begrenzung staatlicher Macht ist Ausdruck eines freiheitlichen Systems, das Freiheit nicht allein in der des wirtschaftenden Bourgeois erfüllt sieht, sondern ebenso der des Citoyens (Kutscha 200I: 22I). 
Da wo die Zusammenarbeit möglich ist, beim Werkschutz, ist sie also nicht wünschenswert, weil der Werkschutz nicht nur nicht an die genannten machtbegrenzenden und Rechtsstaatlichkeit konstituierenden Prinzipien gebunden ist, sondern auch in der Praxis gegen sie verstößt. Man würde den Teufel Kriminalität mit dem Beelzebub Unrechtsstaat auszutreiben versuchen. Dennoch bewegen sich Werkschutz und Polizei in einer Weise aufeinander zu, die Anlass zur Sorge geben muss. Während der Werkschutz, wie oben gezeigt, keinesfalls nur präventive, sondern auch repressive Funktionen erfüllt, führt die Polizei allem Anschein nach die präventive Kehre der siebziger Jahre fort und unterwirft die Bürger einem Generalverdacht, der sich in der Ausweitung polizeilicher Befugnisse verdinglicht. Lauschangriff, Aufenthaltsverbote und Schleierfahndung (vgl. Kutscha 1998) entsprechen nur noch formal dem freiheitlichen Geist des Grundgesetzes; der klassische Mechanismus, erst bei Gefahr einzugreifen, zählt für die Polizei nicht mehr. Allenthalben beklagt man die Auflösung des Störerbegriffs. Der Gedanke, dass der effektivste Kampf gegen Kriminalität darin besteht, Kriminalität erst gar nicht entstehen zu lassen, treibt denn auch tolle Blüten; der Präventionsgedanke sickert in jede gesellschaftliche Ritze ein. Inzwischen wird sogar der Kindergarten als geeigneter Ort für kriminalpräventive Bewußtseinsbildung angesehen. Dass sich in diesem gesellschaftlichen Klima die Rolle der Polizei verändert, braucht niemanden zu verwundern. In einer nach ökonomischen Kriterien geführten Stadt »gerät die Polizei in die Rolle des Garanten von reibungsarmen sozialen, kommunikativen und ökonomischen Abläufen.« (Jaschke I997: 72) Sie übernimmt »die Rolle eines Werkschutzes des Unternehmens Stadt « (ebd.: 73), bei dem die klassischen Prinzipien an Bedeutung verloren haben und Prävention und Repression verschmelzen.

Diese Verschmelzung auf der alltagspraktischen Ebene wird flankiert von einem Ausbau des Gewaltpotential des Staates, das sich zwar vorerst in seiner Anwendung auf Randgruppen beschränkt, aber insgesamt als »Reservefunktion zur Herrschaftssicherung bei zukünftigen Krisen« (Kutscha 200I: 2 I9) zu verstehen ist. Der Abbau sozialstaatlicher Elemente korrespondiert im neoliberalen Staat mit dem Ausbau des Repressionsapparates (vgl. Simon i997), um auf eine ganz spezielle Art den sozialen Folgen der Privatisierung, einer zynischen Logik folgend, zu begegnen. Die privaten Sicherheitsdienste könnten in diesem System, das der sich in zweifacher Hinsicht ausdehnende Staat konstituiert, problemlos integriert werden.

\section{Literatur}

AG Berlin-Tiergarten (1996), in: DAR 65, 8, S. 326-327.

Bauer, Regina (1985): Werkschutz. Seine rechtliche und gesellschaftliche Stellung, insbesondere im Hinblick auf die Bekämpfung der Betriebskriminalität, Heidelberg.

Bundesverband Deutscher Wach- und Sicherheitsunternehmen (BDWS) (2000): BDWS-Stellungnahme zur Gesetzesinitiative, in: DSD 52, 2, S. 3-8.

Britsch, Werner/Königseder, Franz Xaver (1997): Vorschriftensammlung für den Werkschutz und private Sicherheitsdienste, Stuttgart.

Bueß, Peter (1997): Private Sicherheitsdienste. Zur Tätigkeit freier Unternehmer auf dem Gebiet der öffentlichen Sicherheit und Ordnung, Stuttgart u. a.

Busch, Heiner/Werkentin, Falco (1992): Die soziale Produktion polizeilich registrierter Gewaltindizien, in: Polizei-Politik. Streitfragen, kritische Analysen und Zukunftsperspektiven (KrimJ, 4. Beiheft), Weinheim, S. 69-83.

Dixon, Keith (2000): Die Evangelisten des Marktes. Die britischen Intellektuellen und der Thatcherismus, Konstanz.

Ehses, Herbert (1995): Werkschutz, in: Glavic, Jürgen J. (Hg.): Handbuch des privaten Sicherheitsgewerbes, Stuttgart, S. 439-486.

Haupt, Heinz-Gerhard/Narr, Wolf-Dieter (1978): Vom Policey-Staat zum Polizeistaat? Ein Forschungsbericht anhand neuerer Literatur, in: $N P L_{23}$, S. I8 $8-2$ I 8. 
Hoffmann-Riem, Wolfgang (1977): Übergang der Polizeigewalt auf Private?, in: ZRP ı०, I I, S. $277-284$.

Jaschke, Hans-Gerd (1997): Öffentliche Sicherheit im Kulturkonflikt. Zur Entwicklung der städtischen Schutzpolizei in der multikulturellen Gesellschaft, Frankfurt a M./New York.

Jones, Trevor/Newburn, Tim (1998): Private Security and Public Policing, Oxford.

Karazman-Morawetz, Inge (1995): Was macht Stadtbewohner unsicher?, in: Hammerschick, Walter (Hg.): Die sichere Stadt. Prävention und kommunale Sicherheitspolitik (Jahrbuch für Rechts- und Kriminalsoziologie 1995), Baden-Baden, S. 17-37.

KG Berlin (1996), in: DAR 65, I2, S. 504-507.

Kötter, Fritz (1995): Klassische Sicherheitsdienstleistung, in: Glavic, Jürgen J. (Hg.): Handbuch des privaten Sicherheitsgewerbes, Stuttgart, S. 3 I 5-346.

Kutscha, Martin (1998): Große Koalition der Inneren Sicherheit?, in: Bürgerrechte E Polizei 59, S. 57-69.

Ders. (200I): Auf dem Weg in einen Polizeistaat neuen Typs?, in: Blätter für deutsche und internationale Politik, Nr. 2, S. 21 4-221.

Mahlberg, Lothar (1988): Gefahrenabwehr durch gewerbliche Sicherheitsunternehmen, Berlin.

Martin, Joachim (1995): Entkriminalisierungsvorschläge. Beispiel: Beförderungserschleichung $(\mathbb{2}$ 265a StGB), in: Gössner, Rolf (Hg.): Mythos Sicherheit. Der hilflose Schrei nach dem starken Staat, Baden-Baden.

Marx, Gary T. (1987): The Interweaving of Public and Private Police in Undercover Work, in: Shearing, Clifford/Stenning, Philip (Hg.): Private Policing, Newbury Park.

Müller, Rolf (1997): Pilotprojekt zur Überwachung von Kriminalitätsschwerpunkten in der Leipziger Innenstadt, in: Die Polizei 88, 3, S. 77-82.

Ders. (1998): Nochmals: Videoüberwachung von Kriminalitätsschwerpunkten in der Leipziger Innenstadt, in: Die Polizei 89, 4, S. I I4-I I6.

Ders. (2000): Nochmals: Die Videoüberwachung von Kriminalitätsschwerpunkten in Leipzig, in: Die Polizei 91, I0, S. 285 -29I.

Olschok, Harald (2000): Private Sicherheitsdienste in Deutschland, in: Das Geschäft mit der Sicherheit. Tagung der Friedrich-Ebert-Stiftung, Berlin, S. 5 I-60.

Riester, Albert (Hg.) (199r): ASW-Handbuch für die Werkschutz-Fachkraft, Heidelberg.

Roggan, Fredrik (2000): Auf legalem Weg in einen Polizeistaat. Entwicklung des Rechts der Inneren Sicherheit, Bonn.

Schily, Otto (2000): Der Beitrag von Sicherheitstechnik und Sicherheitsdienstleistung zur Kriminalprävention, in: $D S D$ 52, 4, S. 3-7.

Schnekenburger, Franz (1999): Rechtsstellung und Aufgaben des privaten Sicherheitsgewerbes, Köln u a.

Scholz, Rupert (1983): Rechtsfrieden im Rechtsstaat, in: NJW 36, I4, S. $705-712$.

Schroer, Markus (2000): Gewalt ohne Gesicht, in: Leviathan 28, 4, S. 434-45 I.

Senat von Berlin (1999): Sicherheitskonzept für den öffentlichen Personennahverkehr, Berlin.

Shearing, Clifford D./Stenning, Philip C. (1983): Private Security: Implications for Social Control, in: Social Problems 30, 5, S. 493-506.

Simon, Jonathan (1997): Gewalt, Rache, Risiko, in: Trotha, Trutz von (Hg.): Soziologie der Gewalt (KZfSS Sonderheft 37), Opladen, S. 279-301.

Stober, Rolf (I997): Staatliches Gewaltmonopol und privates Sicherheitsgewerbe, in: NJW 50, I 4, S. 889-896.

Ders. (1998): Sicherheit für Wirtschaft und Gesellschaft zwischen Staat und Privat, in: DSD so, 2, S. 28-35.

Wacquant, Loic (2000): Elend hinter Gittern, Konstanz.

Wilson, James W./Kelling, George L. (1996): Polizei und Nachbarschaftssicherheit: Zerbrochene Fenster, in: KrimJ 28, 2, S. I 2 I-I 37.

Wolf, T B. (200I): Die tätowierte Stadt, in: Let's Work, Frühjahr, S. I 3.

Zentralverband Elektrotechnik- und Elektronikindustrie e.V. (ZVEI) (2000): Videoüberwachung Hardenberg-/Breitscheidplatz Berlin. Ein Konzept des ZVEI, Frankfurt a. M. 